\title{
A Cutting Edge Method in Chinese Piano Education: the Xindi Applied Piano Pedagogy
}

\author{
Wei Guo ${ }^{1} \&$ Leonard John Cosaitis ${ }^{2}$ \\ ${ }^{1}$ Xinghai Conservatory of Music International College, Guangzhou, China \\ ${ }^{2}$ Amadeus School of Music, Melbourne, Australia \\ Correspondence: Wei Guo, Xinghai Conservatory of Music International College, Guangzhou, China. Tel: \\ 86-186-6483-4920. E-mail: guowei_judy@ hotmail.com
}

Received: June 3, 2019 Accepted: October 12, $2019 \quad$ Online Published: November 27, 2019

doi:10.5539/hes.v10n1p7 URL: https://doi.org/10.5539/hes.v10n1p7

\begin{abstract}
The Xindi Applied Piano Pedagogy (XAPP) was self-formulated by Professor Xindi, the Dean of the Music Education Institute of Xinghai Conservatory of Music (MEIXHCM), in 2001. After a series of scaffolding developments over a decade, the single instrumental teaching method is now developing into a holistic music educational system for the preparation of graduate music teachers at both primary and secondary levels. In addition, this particular pedagogy seems to have created a competitive force in the market of community music education in mainland China. This article explores the XAPP in a narrative manner whilst investigating the advantages it has over three classical repertoire-oriented piano methods in mainland China in the modern era.
\end{abstract}

Keywords: Xindi Applied Piano Pedagogy, mainstream piano methods, interest cultivation, accelerated learning, mainland China, practicability

\section{A Scope of Piano Education in Mainland China}

Since the popularization and development of music education in modern times, many children in mainland China are able to get access to advanced and quality music education at a very young age. Among all types of musical instruments learning, piano seems to have received a great deal of preference (Cui, 2012; Pan, 2018; Wang, 2019). Upon the inception of the "Opening-up" policy in 1978, piano learning in mainland China soon developed into a thriftily growing socioeconomic phenomenon emanating from the imported Western culture. As early as 2005, Wu (2005) had already cited Liu Shi Kun as one of the most prominent contemporary Chinese pianists, confirming that "China has become the world's most rapidly rising [consumer of] piano education" (p. 4).The statistics collected by The Chinese Musicians Organization (CMO) stated that by the end of 2013 the population of piano learners in mainland China had exceeded 30 million (CMO, 2019). Liu (2009) adds that from the early 80 s of the 20th century to 2009 , over 200,000 piano learners had successfully entered tertiary institutions for further professional piano studies every year. Moreover, China Industry Information Network (CIIN) reports on their official website in 2015 that in the year of 2010 China produced 358,500 pianos, taking 73.33\% of the entire Asian production (CIIN, 2019).

Conversely, the piano learning market in recent years in mainland China is experiencing stagnation, with particular deterioration in the metropolises. From the author's observations, Chinese piano education has plunged into three materialistic pursuits of objectives, including certificates of graded-examinations, trophies of competitions and mono-dimensional developments in techniques. Wei (2019) believes that what sacrificed the most in such types of piano teaching is musicality and that the deficiency in musicality has resulted in the quasi-equality between piano education and technical training. The artistry and cultural contributions that piano education emanated from is long gone. This understanding is supported by Liu (2009), who asserted that "It is easy for children to lose their interest in piano and in music simply by studying for purposes such as exams. The parents will only be paying more attention to their children's next grade rather than the children's aesthetic development nor the sustained enjoyment in piano playing. All this is because this type of teaching has become a kind of thinking pattern" (p. 29).

Based upon many years of professional learning and teaching experience, Wang, as a sophisticated piano educator for amateurs, finds that some long existing problems in China's piano education are critical and have 
never been resolved in any form or fashion (2019). She points out that piano training for amateurs generally starts with the good will of the learner but very often ends with the learner's dissatisfaction in piano and, worse, in some cases, in music per se. Wang blames the lack of interest-centered mentality among a great number of piano teachers for this dramatic change (2019). She further discloses that piano teachers who have little concern about cultivating the learners' interests during their teaching 'business' usually have insufficient or, alarmingly, zero music-education qualifications. Wang believes that, disappointingly, teaching piano to the "pirate" teachers is a means of making money which is no different to killing a piano learner's musical life (2019).

Compared to Wang's teacher-oriented understanding of the problematic status of piano education in today's mainland China, Pan's (2018) observation seems to be more comprehensive. Firstly, he is in agreement with the fact that the uneven quality of piano teachers is one of the major causes. Secondly, Pan (2018) points out that other causes that might prevent the positive development of Chinese piano education are manifested in single-dimensional teaching methods, the lack of innovation in teaching mentality, the poor training qualities in community piano institutions, the disadvantage in academic research in piano education, as well as the faulty evaluation system for qualifying piano teachers at different levels.

From the authors' perspective, the emergence of the aforementioned problems from the past to the present piano education in mainland China has its roots in historical and social aspects. Such problems are inevitable in the development of piano education in consideration of other difficulties associated with the development of the entire country, especially in an age of marketing-oriented arts education. Despite the fact that a number of extraordinary pianists, including worldly renowned Lang Lang and Wang Yujia, being cultivated within the adapted western music culture, only a few among the rest aspired to become professional pianists. Most of the learners entered the piano world either self-motivated or under their parents' directives, merely to acquire a musical hobby or some skills to entertain themselves and others on the keyboard. Thus, interest is rooted in almost every piano learner's learning motivations. To the authors, any successful piano teaching method that could survive the many problems in piano education as mentioned above should be characterized in terms of practicality, and the core of practicality in this context is the cultivation of the individual learner's interest in piano as well as in music.

\section{Three Mainstream Piano Teaching Methods and Their Associated Problems}

\subsection{The Teaching of Graded-examination}

Many would argue that studying for graded-examinations is the ultimate demonstration of being practicable. The authors' response to such opinion is, however, ambivalent. Liu Xiao Long, an expert in the history of Western music and an associate professor at Peking University, published a series of ten articles about The Sixty Years of Chinese Piano Art between 2009 and 2010. In the ninth article, Liu (2010) described clearly about the inception of graded-examination system in mainland China. In the mid-1980s, some of the elite musicians had generated the idea of evaluating piano students on their performing abilities. Such an idea came from their observations on the examination activities conducted by The Associated Board of the Royal Schools of Music (ABRSM). The first official graded-examination system was not launched until 1991 and was developed by the Chinese Musicians Organization (CMO) to standardize the growing music teaching market and give timely evaluation and guidance to the vast body of piano learners outside the music conservatories. For the first time, piano teaching across mainland China achieved a unified evaluation standard. In Liu's view, for both teachers and learners, the graded-examination provides a quantifiable standard for accurately evaluating each individual's learning progress and the learner's actual playing ability, whilst clarifying the goals and directions for future learning (2010).

In many of the academic articles the authors collected and analyzed, the advantages of piano graded-examination system are obvious and broadly recognized. For example, Lv and Liu (2017) in their research on the ABRSM examinations, conclude that this particular examination system encourages piano learners to acquire comprehensive musicianship, especially music theory and aural abilities. Yin and Tang (2018) found in their investigations that when studying for the graded-examinations, the learners get the opportunity to constantly examine themselves by detecting their frequently made mistakes and correcting themselves. On the other hand, the disadvantages of graded-examination system have demonstrated an equal, if not greater impact on piano education in mainland China. Yang (2014) reports that graded-examination has become the sole learning purpose for many piano learners, a tool for parents to compare their own child with others, and the best excuse for some piano teachers to practise "lazy teaching" whilst earning significant income.

All these phenomena have demonstrated a significant departure of piano education from the original intention of cultivating learner's sentiment. Wang (2012) adds that in order to master the set pieces for the examination, the 
learners need a long time to prepare. With the limited time on piano practice for many piano learners, practice on the set pieces takes up their entire piano time. Consequently, many learners have absolutely zero understanding of the background knowledge of the set pieces. In this regard, Wang (2012) believes that the graded-examination works no differently than having the effect of confining the learner's musical path, ultimately leading to limited musicianship.

The authors' critical evaluation of the graded-examination system renders the observation that the system should play different roles in accordance with each individual learner's particular learning needs and should never become the sole motivation and/or final destination for piano learning. In other words, the graded-examinations system is only "practicable" when it becomes a part of the interest cultivation of the learner's piano learning process. Furthermore, the practicability in piano learning is to serve the purpose of providing an interest-oriented and comprehensive musicianship for the learner. Passing examinations grade after grade makes piano learning "practical" but only for the activity of piano learning, rather than for the actual subject of this activity: the learner herself.

\subsection{The Teaching of Competitions}

Teachers without exam-driven mentalities are not necessarily advocates for interest-oriented and user-friendly practicality in piano education. To some piano teachers, the word "practicality" is believed and interpreted in their piano teaching by directing students towards preparing for competitions from the outset. That is to say, instead of taking up the complete piano teaching process with graded-examinations, such teaching mentality delivers nothing else but piano competition preparations to the students. In terms of the characters, standards and qualities of the competitions, they vary from officially organized international ones to privately arranged party-like ones. Consequently, it becomes a commonality that some piano learners who have been continuously "learning" piano for a few years and are "championship winners" can only manage to play a very limited repertoire with extremely inadequate technical skills and close to zero knowledge or understanding about the pieces they play throughout their entire learning experience.

Huang (2017) observes that due to the marketization of piano education, the commerciality of piano competitions is becoming stronger and stronger, and the number of people who have won gold or first prize far exceeds the proportion. It is of little significance to participate in such a competition. If the child does not prepare carefully or is not fully prepared, but gets a good ranking, it is easy for the child to be blindly confident and get lucky, thinking that he or she does not need to make a lot of efforts to get a good score. Therefore, parents and teachers must be careful to choose such games. In addition, due to the need to put more energy into the competition, for the children who lack playing ability and practice the piano with low efficiency, the competition will affect their normal learning progress to a large extent.

It is also common to see students quit piano after making a couple of attempts in the competitions simply because such type of piano learning, which is more like a trophy manufacturing process to the authors, is believed to be completely dull, mechanical and mission-driven. Some learners suffer from serious performance anxiety and, therefore, participating in competitions as a soloist could be mental threats to them. In an investigation in Chongqing (one of the major cities in mainland China) children's piano music education, Wang (2014) concludes that it is facing many problems, and encouraging if not forcing young learners to participating in the chaotic piano competition filed is one of the major problems. He concerns that if not solved in time, Chongqing children's piano music education will be deviating from the quality education, aesthetic education track, into the wrong path of utilitarianism, and ultimately restrains its development (Wang, 2014).

From the authors' perspective, both graded-examinations and competitions in piano education are meant to be utilized as alternative methods for optimizing one's regular piano learning and could prove extremely beneficial if appropriately positioned in one's piano learning process. However, once they take up the entire learning process, piano education loses its elemental meaning, and is no longer practicable in terms of developing one's holistic musicianship.

\subsection{The Teaching of Mono-dimensional Techniques}

The aforementioned two groups of piano teachers who conduct their entire teaching through preparing students for exams and competitions are not minorities in mainland China in present days. As for the rest of the piano educators, not all of them are able to structure and deliver their teaching practice, implement their teaching methods and select teaching materials for the purpose of stimulating and/or nurturing their students' interest. A great number of piano teachers simply and rigidly follow the mono-dimensional systems which they were trained under as far as several decades ago. Such teaching mentality solely focuses on techniques without any adaptations to the latest developments in the music field or any additional teaching methods to address students' 
needs in practicality.

The technique-driven traditional teaching mentality can be easily detected in the teaching materials the teacher chooses for all of his or her students from the first day of the teacher's teaching career to the present day. The traditional teaching materials usually consist of technical works, finger exercise, etude collections in sequence and sonata collections in volumes. The selecting range has been set for several decades and the repertoire lays a strong focus on western classical music. Many of the traditionalists only use this series of teaching materials without any additional repertoire and never introduce their students to improvisations and singing-playing activities. Under this type of technique-centered piano instructions, students lose their interest in piano and eventually in music very easily as the repertoire is very distanced from their daily life and the learning process is completely dull. This type of traditional teaching in music education is not practicable whatsoever and is capable of limiting the learner's development in a comprehensive musicianship.

Zhang (2017) inserts that for the elementary piano students, the technique-centered piano teaching spares the experiential feelings during the learning process, delivering a concept of passive learning which can easily result in the student's loss in interest and possibly cause the student's departure from the emotions obtained by the senses towards music. Ten years before Zhang's observations, Gao (2006), from her teaching experiences, had already reported that many parents and teachers devote most of their children's piano learning time to practicing techniques in conjunction with a very limited repertoire, ignoring the improvement of children's overall cultural and artistic quality. As a result, they could learn nothing from the music because of the detachment from the rich artistic resource and the broad spiritual vision that the nature of piano learning offers.

All in all, learning piano for succeeding in graded-examinations, collecting trophies through participating in numerous competitions and or training superior technical skills forms the general understanding and interpretation of practicality of piano education among the majority of the educators and learners. To the authors, these mono-purposed piano activities have misplaced their focus in piano education. Instead of focusing on the learner, the subject of the learning process, they center the process on objectives with the belief of being "practicable". It is admitted that any piano learning process and outcomes need to be evaluated for what has been learnt and what to be learnt next. It is also agreed that taking graded-examinations and participating in various type of competitions are ideal means for the evaluation no both teachers and learners. However, all types of evaluating methods are meant to be utilized and practised periodically throughout one's piano learning process and only for the purpose of enhancing the learning in general. One thing must be clarified is that both learning and evaluating are to serve the subject of the learning activity, the learner, in accordance with the development of the subject's comprehensive musicianship.

It is assumed that any piano teaching method which deviates from interest culmination and user-friendly practicality may cause intrinsic damage to piano learners, especially the younger ones' perceptions towards music in general. Thus, in order to enable a healthy and positive morale in the field of piano education, educators must give priorities to interest cultivation and user-friendly practicality. Eventually, the loss of interest has drawn significant attention from parents and, at the same time, the practicality of many existing piano teaching methods are urged to be reflected upon. Consequently, maintaining and developing student's interest in piano learning has become the priority for many piano educators, especially in present days. It is also believed that interest-oriented teaching methods with particular emphasis on practicality should make a return to the field of elemental piano education in mainland China.

\section{About the XAPP}

The Xin Di Applied Piano Pedagogy was officially established in 2001 by Professor Xindi at Xinghai Conservatory of Music in China. According to the latest official annual report provided by the Xindi Music Academy (XDMA), the current practice on the XAPP has three major interdependent divisions, including: (1) open-teaching for professional piano players, music teachers and amateurs at various music schools in different regions across mainland China; (2) tertiary education for all Music Education majored students at Xinghai Conservatory of Music, Guangzhou, China; and (3) overseas franchised training at collaborative local music schools in America and Australia. Professor Xin describes his self-invented piano teaching method as revolutionary. With the teaching philosophy of "happy piano learning, interest-based piano learning, accelerated piano learning, and creative piano learning", the XAPP has clearly placed priorities on cultivating the learner's interest, creativity and collaborative abilities. It is imperative that the pedagogy is conducted through a repertoire that is closely related to the learner's daily life in order to optimize the learner's particular music preference through piano learning.

62 textbooks have been published and are utilized to address various essentials for learners at all levels. 
According to different learners' underlying learning purposes, Professor Xin has categorized his 62 books into 12 series, including (1) piano education for early childhood; (2) piano education for school children; (3) piano education for adults; (4) piano foundations; (5) piano performance; (6) singing-playing with piano; (7) piano for four hands; (8) piano accompaniment; (9) transpositions on piano; (10) piano composition; (11) piano harmony; and (12) Jazz piano. The complete set of my books is featured in its comprehensiveness, flexibility, variety and practicality. Out of all these features, the fundamental core is the capability for motivating, cultivating, activating, developing, and preserving both teachers' and learners' interest in music. So far, over 30 thousands enthusiasts have followed this pedagogy, including music teachers, piano learners and armatures from all over the world.

In the 62 books that Xin has written for XAPP, theoretical fundamentals are introduced to piano learners as early as possible but in a practicable manner. It is further explained in Xin's educational theory that being practicable requires the teaching method to include an easy-to-follow structure, succinct instructions, a broadly-ranged repertoire, various intuitional examples, and a sufficient provision of progressive exercises in correspondence to each learning point. In the XAPP, the singing-playing skill is regarded as its key feature and has been included and emphasized for learners at all levels. The authors realize that many piano players who are trained under mainstream methods find it is rather challenging to just physically manage the coordination between singing and accompanying and, therefore, improvisation involving singing-playing becomes virtually impossible to accomplish. Professor Xin noticed this common hurdle via his observations throughout the many years of his teaching and supervising. Hence, in the XAPP, singing-playing learning is placed as a key point throughout the entire process for all learners, not only for the talented ones.

\section{The Essence of Xin's Educational Theory}

One of Xin's educational perspectives is that in this internet scaffolding era of the 21 century, knowledge and skills as a form of digital mechanism can be accessed anywhere, at any time within the "net" field. However, "true knowledge" from his understanding is not only what to see, what to hear and what to memorize but also what to be applied. In other words, the knowledge is only "true" when it is practicable and valued by the society where it is conceived. This is why the XAPP is becoming worldly recognized and is receiving a growing number of invitations from abroad for adoption as a practicable and society-valued pedagogy.

\subsection{Practicability and Value}

When talking about education, the authors believe that it is imperative for the term to be interpreted contextually, on its own, but never in isolation from the culture where the particular form of education is cultivated in conjunction with the people who conduct the educational activities. The nature of this article is to examine the XAPP's justification in the field of piano education in today's mainland China. Under such contextual scope, the authors focus on the underlying core of the XAPP's practicability to discuss whether this particular pedagogy is a cutting edge revolution or just anther ephemeral phenomenon. From the perspective of both authors, the educational theories of John Dewey in Pragmatism is considered the most suitable theoretical platform for analyzing and discussing the practicality of the XAPP.

On the basis of his philosophical, sociological and psychological background, Dewey extended his pragmatic view into the field of education. In the $20^{\text {th }}$ century, the inspection of Dewey's Pragmatism Education signified the establishment of the "modern pragmatism education system" (Xiao, 2011). In general, Dewey's educational pragmatism encompasses the following aspects:

(1) The essence of education. In his theory of education, Dewey discussed the essence of education from multiple angles. His views on the nature of education can be basically summarized by the three important arguments which he put forward, stating "education is growth", "education is life" and "education is the continuous transformation of experiences".

(2) The purpose of education. Dewey discussed the purpose of education in many ways, but the most important one was his paradoxical statement that "education has no purpose".

(3) The fundamental principle of teaching. The core of Dewey's theoretical system of education is his teaching theory, and "learning by doing" is the fundamental principle of his overall teaching advocacy.

Not unlike aspects of Xin's XAPP, following suit more than 150 years later, Dewey discussed education from the perspective of the practicability in education. From the authors' point of view, Xin's advocacy of practicability in piano education is comparable with Dewey's Pragmatism in education in many ways, but in this article the commonalities they share are interpreted in accordance with two particular aspects, including the practicability and the value of education. 
Education should center on its practicability and its value orientation. In terms of piano education, Xin realizes that the majority of the young learners who are trained under mainstream methods only practise for two reasons, i. e. collecting certificates or trophies, and improving their playing techniques to please their teachers and parents. Consequently, after making a few attempts in the exams and achieving a couple of trophies they refuse to continue the learning. Some learners can only manage to play a maximum of five pieces of music after reaching the top grade of the exam system because that was all they had learned and practised throughout their entire learning experience. Xin regards this type of piano learning as nothing but a burden to the learner, both physically and mentally.

Apart from a very small population of the learners who have strong interests in appreciating classical music and applying what they have learned in their daily life for entertainment and satisfaction, the rest only learn to forget what was learned. The reason is that most of the mainstream piano teaching methods lack "usefulness" in the learner's daily life in current times. To Xin, the value of music education should be generated from its usefulness in entertaining, relaxing and satisfying the learner him/herself through systematic and professional learning experience with simply structured, quickly grasped, less technical, self-rewarding and creative methods. According to Xin, most of the young learners of the XAPP are able to play their interested music immediately after they hear from the media. What he means by "music" in this context is not only the melody, but also self-composed and improvised accompaniment. This ability for most of the young learners who are trained under the mainstream methods appears to be challenging, if not overly-difficult, unless all the music is clearly notated and practised a number of times depending on his or her sight-playing abilities. On top of the efficacy, what is more exceptional is that with the XAPP, parents are set free from pressuring their children to practise on the keyboard and, in most cases, parents are not even interested in the classical repertoires their children practise. Under the instructions of the XAPP, learners are allowed to select their favourite tunes to practice, improvise and even accompany whilst singing. Most of their favourite tunes are child-friendly, popular and easy to sing along to and, therefore, are favored by their parents and peers. Consequently, practising on the piano is no longer a "duty" to fulfill just to please the parents or in preparation for exams, but something that relaxes, entertains and rewards the learner him/herself after a day of intensive school work.

\subsection{Revealing of Authenticity}

Education should aim at revealing its authenticity. In Xin's case, the authenticity of education is interpreted as the practicality of what's learned from and for life-experience for people of any age group, including both instrumental and non-instrumental education. Xin opposes most of the mainstream piano teaching methods that have been dominating piano education in mainland China for many years. He finds that those types of teaching mainly base their practices on their training students to become instrumental mechanics through utterly dull technique repetitions with a rigid repertoire of classical music only. It is obvious to Xin that both the teaching philosophy and the teaching materials of those mainstream methods no longer fit into people's present lifestyles, music appreciation versatility, time management disposition, and so on. All in all, they seriously lack practicality as they are not tightly bound with people's daily lives in present times and the learned techniques and compositions are not practical enough to support one's personal musical needs other than piano learning, such as playing favourite tunes with ultimate chords as accompaniment, improvising at the piano to address different entertaining scenarios, or being able to compose on the piano even at the early stage of the learning process.

In terms of revealing the authenticity of education, Xin further argues that most of the mainstream piano teaching methods have been proven to be considerably impracticable and comparatively mono-functional over the decades by a great number of learners who have quit piano learning after investigating numerous case studies over a number of years. He continues to advocate that piano education should involve the training of one's singing, playing and improvising skills together, not separately, from the very beginning of his or her learning process. However, the structure of the teaching method should begin with the simplest concepts and easiest exercises; a gradual cultivation of sequential learning experiences which encompass singing, playing and improvising resulted from Xin's advocacy and in his twelve models of teaching materials. Xin is in concordance with Carl Orff's insistence that "ideally, piano learning should begin in early childhood, and should make use of the child's own musical experiences as materials for the instructional process" (Landis \& Carder, 1972).

Xin accepts the importance of elemental learning in one's entire musical life, however, he adds that many piano players who are now in their middle and advanced ages demonstrate strong interest in making a start in piano learning merely because it was not possible to achieve in their childhood for various reasons. Among the twelve models of the XAPP, Model 3 which is clearly named as Piano Program for Adults, is specifically designed for adults with nil technique foundation and are pathetic in music literacy. Xin structured this program within ten lessons for what he calls "accelerated learning" in consideration of adult-learners' limited time allowance for 
both learning and practising. Out of the ten chapters in Model 3, each chapter centers on one or two popular music works, catering for the learner's (1) finger positions on the keyboard; (2) numerical notations and harmony marks; (3) elementary rhythmic patterns; (4) basic pedaling skills; (5) commonly used accompaniment patterns; and (6) simplified improvisational skills.

Since the establishment of the premiere institute of the XAPP in 2001, there have been over forty divisional schools launched over the world. These teaching bases are located in different cities in mainland China as well as in overseas. Three hundred and eight workshops for music teachers have been conducted in 48 cities of mainland China to the present day and 48 examination centres have been launched nationwide. The music teachers who have participated in the workshops have applied the XAPP into their daily teaching not only to those who have learnt under mainstream methods, but also to amateurs, including elderly persons, full-time housewives, business people, etc. Many XAPP learners have agreed that playing piano through this accelerated method has made a significant change to their lifestyles. Those who have mainstream piano learning background are now freed from music scores and are able to play by ear, whilst those who have zero music background are able to play their favourite tunes with simple improvisations as accompaniment.

\section{Conclusion}

Piano education is the music education with piano as the media, and its teaching practice is inseparable from the key factors of contents, objectives and outcomes. At present, the discussion centering on how to make the diversified integration between piano education and mass culture more in line with the development of China's modernism has become a topical issue of strong focus in the field of music education. Based upon the demonstrations and analyses of the three dominating phenomena, i.e. examination-driven, competition-driven and technique-driven teaching fashions and their corresponding problems over the past few decades of piano education in mainland China, this paper has critically discussed the characteristics of the Xin Di Applied Piano Method as an innovative method in the present era of modern piano education. The authors have taken a clearly defined perspective in depicting the XAPP as a practicable, innovative method by dint of its simplified, interest-cultivating, integrated and creative features, which cater to the public at all age levels.

XAPP's practicability in a self-feeding loop of learning is able to enhance the overall accomplishment of piano playing. Learners are, hence, gratified and further motivated with growing levels of piano-playing skills, gauging their own acquisition through increased and elevated practicability. Being 'learner-friendly', the XAPP's merits are also enhanced in its orientation as being practicability-driven, rather than exam-driven. Notwithstanding its many advantages, the XAPP, will never fully supplant mainstream piano-playing pedagogy (just as American-English, arguably, will not supersede British English, despite its overtly systematic simplification of spelling). The XAPP, however, does offer an alternate and simplified language to music learning that will reach myriads of enthusiasts. These avid learners will be those students who are prepared to take the bold step of cutting the Gordian knot towards mastering piano playing in a much-reduced time-frame.

Not only that, but also in the XAPP's teaching process, Xin demolished the mindset of "building the foundation for music application" as believed by many mainstream piano teachers, breaking away from the traditional thinking manner. Innovation is the force for improvement, upon such mentality, Xin has paid a lot of attention to common practice and at the same time, integrating the development of the learner's personality into the learning process. In order to do so, it is necessary to understand the learner's musical appeal and interest points respectively. The learner's music preference is another focal point in the learning process. Whether it is pop music, folk songs or screen music or even video game music, the learner is only fully engaged in the learning process when he or she is learning to play the music that is related to their daily life as well as can create a common ground for the learner's audience in real life.

In terms of comprehensive musicianship, not only music theory, aural training are included in the learning process of the XAPP from the very beginning but also composing and harmony. Xin advocates for such an integrated method because it is beneficial in helping the learner realize the diversification of music notation, forms and styles from the outset. It is not uncommon to include music theory and aural training in traditional teaching methods or mainstream piano education, but there is hardly any incorporation of teaching of composition and harmony throughout the entire teaching process, leaving alone placing such learning components at an early stage. On top of this, the XAPP has also abandoned the so-called disadvantage of only focusing on the "vertical" depth of a certain module in traditional piano teaching. Instead, it paid a lot of attention to the "horizontal" connection in the piano teaching module, so as to enable learners to maintain a sustained interest in in-depth learning and exploration step by step.

Professor Xin truly realized that only localization can make the piano, an exotic western instrument, to gain 
vigorous vitality in this country in the modern era. With particular considerations of the characteristics of Chinese piano education market, Professor Xin has been exploring and taking on board excellent foreign educational methods over the years and inheriting and carrying forward the advantages of Chinese traditional educational concepts. He hopes that every learner can express their feelings with the piano and express their feelings with the music their daily life is set upon. His educational theory of "anti-archeology" in piano learning has formed the foundation for the establishment of the XAPP, placing emphases on cultivating the learner's' sustained interest throughout his or her entire piano learning journey. The future development of the XAPP is aiming to promote the "applied piano education" for the mass, while inheriting the traditional Chinese music education for the young generations. Xin hopes that his theories in music education can break the long existing dominance of the mainstream teaching methods, and create a new age for China's "applied education" in all music associated areas.

The Xindi's Applied Piano Pedagogy is widely applied to people of all ages and various professions across the country. It is advocating to the public that piano learning is meant to enhance the quality of the learner's daily life and shall not to force the learner to become a "piano archeologist". The XAPP is making every possible effort to make piano learning into ordinary people's life, helping ordinary people realize their piano dreams of "free to play". "The performing stage belongs to every individual and the music belongs to the public." says Xin. Whilst advocating that every individual should be able to play piano without being constrained by techniques, the XAPP also reinforces its core belief in what is learned must be put into practice in order to serve the learner's life. As the featured component of the XAPP, the singing-playing skill helps the learner more than any other skills in terms of making piano learning closer to people's life. Basing upon his analysis of the current situation of piano education in China, Xin concludes that piano learning shall never be equalized with "piano archeology", but an ultimate method to develop each individual learner's comprehensive musicianship with the aim of becoming a lifelong self-motivated music "ambassador" between the past and present, and, the self and the world.

\section{References}

Barnacle, R. (2001). Phenomenology \& Education Research. Retrieved from http://www.aare.edu.au/01 pap/bar01601.htm

Bourke, P. E. (2007). Inclusive education research and phenomenology. Australian Association for Research in Education Conference. Research impacts: Proving or improving?

Chen, X. X. (2017). On the innovation of "applied piano teaching method" in piano enlightenment teaching. Dramatist.

Chinese Musician Association (2013). Retrieved from http://www.chnmusic.org/cmanews.html 12/05/2019

Chinese Industry information network (2015). Retrieved from http://www.chyxx.com/industry/201510/351654.html

Cui, Q. G. (2012). The misguides and corrections of current piano education. Journal of Mianyang Normal University, 6, 136-139

Ding, F. F. (2019). Reflections on Foundational Piano Education in China in the New Era -- based on the inspiration of the development and evolution of piano education in the past three hundred years. China music, 2, 154-160+178

Huang, J. Q. (2017). Influence of participating in piano competition on children's piano learning. Art \& technology, 30(01), 403.

Liu, X. L. (2009). China piano art development 60 years (four). Piano art, 03, 26-28.

Liu, X. L. (2010). China piano art development 60 years (9): piano grade examination in the learning boom. Teaching Research, 01, 26-28.

Liu, X., \& Lu, T. M. (2017). Advantages and disadvantages of ABRSM piano grade examination in China's Society Music Education. Piano Art, 10, 40-44.

Pan, H. Y. (2018). On the status quo and countermeasures of piano education in China. Popular Literature and Art, 22, 230.

Reid, K., Flowers, P., \& Larkin, M. (2005). Exploring Lived Experience. The Psychologist, 18(1), 20-23.

Tao, F. R. (2016). An analysis of the "pragmatism" phenomenon in contemporary Chinese piano education. Hunan Normal University. 
Wang, X. (2012). Advantages and disadvantages of piano grading examination in universal piano education. Literature and Education Materials, 16, 106-107.

Wang, Z. (2014). Research on Chongqing children's piano music education status and development. Chongqing Normal University.

Wang, Y. (2019). Research on community piano education. Journal of Inner Mongolia Radio and Television University, 02, 30-32.

Wang, H. L. (2019). Analysis on the status quo of piano education in China. Art review, 08, 103-104.

Wei, P. (2019). Vertical and horizontal comparison and reflection of Chinese contemporary piano education. Sichuan Drama, 03, 140-143.

Wu, D. M. (2005). Liu Shikun on music education. Primary and secondary school music education, 06, 4-5.

Wu, D., Guo, L. W., \& Luo, C. (2019). Theoretical thinking on the balanced development of piano education in China from the perspective of culture. North China Music, 3, 186-188.

$\mathrm{Wu}, \mathrm{Y}$. (2017). Research on the current situation of piano education in contemporary China. Dramatist.

$\mathrm{Xu}, \mathrm{H}$. Y. (2006). From skill training to quality improvement -- a case study of returning to the essence of piano education. Inner Mongolia Education, 07, 32-33.

Yang, Y. M. (2014). Learning piano does more harm than good in grade examination. China Education Daily.

Yu, X. J. (2011). Post-philosophy culture-based school music education. Nanjing: Nanjing University.

Zhang, L. L. (2017). Exploration on experiential teaching method in primary piano teaching. Northeast Normal University.

\section{Copyrights}

Copyright for this article is retained by the author(s), with first publication rights granted to the journal.

This is an open-access article distributed under the terms and conditions of the Creative Commons Attribution license (http://creativecommons.org/licenses/by/4.0/). 